\title{
SIC-MMSE Method based Wireless Precoding Technique for Millimetre-Wave MIMO System
}

\author{
Khaizuran Abdullah and Rounakul Islam Boby* \\ Kulliyyah of Electronic and Computer Engineering, International Islamic University Malaysia, 53100, Gombak, \\ Kuala Lumpur, Malaysia; khaizuran@iium.edu.my, rounaqul2020@gmail.com
}

\begin{abstract}
Objectives: A communication method is proposed using Minimum Mean Square Error (MMSE) precoding and Successive Interference Cancellation (SIC) technique for millimetre-wave multiple-input multiple-output (mm-Wave MIMO) based wireless communication system. Background: The mm-Wave MIMO technology for wireless communication system is the base potential technology for its high data transfer rate followed by data instruction and low power consumption compared to Long-Term Evolution (LTE). The mm-Wave system is already available in indoor hotspot and Wi-Fi backhaul for its high bandwidth availability and potential lead to rate of numerous Gbps/user. But, in mobile wireless communication system this technique is lagging because the channel faces relative orthogonal coordination and multiple node detection problems while rapid movement of nodes (transmitter and receiver) occur. Methods/Improvement: To improve the conventional $\mathrm{mm}$-wave MIMO nodal detection and coordination performance, the system processes data using symbolized error vector technique for linearization. Then the MMSE precoding detection technique improves the link strength by constantly fitting the channel coefficients based on number of independent service antennas (M), Signal to Noise Ratio (SNR), Channel Matrix (CM) and mean square errors (MSE). To maintain sequentially encoded user data connectivity and to overcome data loss, SIC method is used in combination with MMSE. Improvements: MATLAB was used to validate proposed system performance. Simulation analysis has shown that, with the increase number of antenna use, the spectral efficiency also increased and higher then millimetre-wave MIMO or Single MMSE system. This research observed that, hybrid controller or combined control method have the better efficiency then single method, where SIC-MMSE based hybrid controller is a good example.
\end{abstract}

Keywords: Channel Matrix, Long-term Evolution, Millimetre-wave, Minimum Mean Square Error, Quantized System, Successive Interference-Cancellation, Wireless Communication

\section{Introduction}

The millimetre wave technology in communication system still in progress mode, where it is fall behind because of having exponentially increase number of path losses, high shadow fading, requires high power to overcome blockage and the most important is penetration losses. Where this system also leading some other issues to have poorer link margin than legacy systems $\frac{1-3}{}$. The millimetre wave also uses spatial sparsity for the channels with large antenna array system with physical layer beamforming for directional transmission of signals. Many researchers have done their studies on the design and analysis the performance of the beamforming directionally as well as system precoding for best Multi-Input Multi-Output

*Author for correspondence 
(MIMO) performance ${ }^{4-7}$. In practice, apart from the small cell coverage, it's also possible to increase array gains in case of using large antenna arrays for the long-distance communication; a significant improvement can be found. Researchers also discover that directional array is good for fast data transfer standpoint and robust to phase changes across clusters and allow a smooth trade-off between peak beamforming gain and initial user discovery latency. There has also been progress in generalizing such directional constructions for multi-user MIMO transmissions $s^{\frac{8-11}{}}$. Researcher proposed several types of hybrid beamforming or array system for wireless millimetre technology. They combined the solutions of the millimetre wave limitations with optimal precoder technique and controlled with hybrid architecture addressed as a sparse reconstruction solution ${ }^{12}$, where basis pursuit method was used for the algorithms and solutions. The combined hybrid solutions shown some good performance, where the performance gap of the proposed systems addressed in the reference paper $\frac{12}{}$, and large array structure with an iterative method proposed in $\frac{13}{3}$ and adaptive estimation according to hierarchical training codebook for the channels shown in ${ }^{\frac{14}{4}}$. Performance comparison with previous works and result that, with the little addition of iteration schemes are enough for optimal performance. Some researcher proposed and successfully found that, when they combined hybrid system in digital architecture and twice the number of data-streaming RF channels, the performance dramatically increases ${ }^{15}$. A heuristic algorithm with good performance is developed when this condition is not satisfied. Several other works such as ${ }^{16,17}$ have also explored iterative/algorithmic solutions for hybrid beamforming. A common theme that underlies most of these works is the assumption of phaseonly control in the RF/analogue domain for the hybrid beamforming architecture. This assumption makes sense at the user end with a smaller number of antennas (relative to the base-station end), where operating the PAs below their peak rating across RF chains can lead to a substantially poor uplink performance. On the other hand, amplitude control (denoted as amplitude tapering in the antenna theory literature) is necessary at the base-station end with many antennas for side-lobe management and mitigating out-of-band emissions. Further, given that the base-station is a network resource, simultaneous amplitude and phase control of the individual antennas across RF chains is feasible at millimetre wave base-stations at a low-complexity and cost $\frac{18}{18}$. The millimetre wave experimental prototype demonstrated in allows simultaneous amplitude and phase control as shown in Table 1.

Table 1. Summarization of notable review papers

\begin{tabular}{|c|c|c|c|c|c|}
\hline Methods & Years & \multicolumn{2}{|c|}{ Advantages } & \multicolumn{2}{|c|}{ Disadvantages } \\
\hline $\begin{array}{c}\text { Network feedback } \\
\text { based IOT }\end{array}$ & 2014 & & $\begin{array}{l}\text { connect, control } \\
\text { and monitor more } \\
\text { than one appliance } \\
\text { together through } \\
\text { IoT. }\end{array}$ & 1. & $\begin{array}{l}\text { Constant monitoring } \\
\text { and services are } \\
\text { required. }\end{array}$ \\
\hline $\begin{array}{c}\text { Wired Building } \\
\text { Automation }\end{array}$ & 2015 & & $\begin{array}{l}\text { pilot models with } \\
\text { the wired network. } \\
\text { Web-based } \\
\text { instrument. }\end{array}$ & & $\begin{array}{l}\text { Maintenance } \\
\text { required. } \\
\text { Automatic nodal } \\
\text { updates not possible. }\end{array}$ \\
\hline
\end{tabular}


Table 1 Continued

\begin{tabular}{|c|c|c|c|c|c|}
\hline $\begin{array}{c}\text { Network-based FDS via } \\
\text { CAN }\end{array}$ & 2004 & $\begin{array}{l}1 . \\
2 .\end{array}$ & $\begin{array}{l}\text { Very short } \\
\text { response time. } \\
\text { Direct data } \\
\text { accusation for } \\
\text { accuracy. }\end{array}$ & 1. & $\begin{array}{l}\text { Data received } \\
\text { directly from the } \\
\text { fire detectors any } \\
\text { interruption means } \\
\text { system loss. }\end{array}$ \\
\hline $\begin{array}{l}\text { FPGA based smart } \\
\text { system. }\end{array}$ & 2016 & $\begin{array}{l}1 . \\
2 .\end{array}$ & $\begin{array}{l}\text { Smart system. } \\
\text { Auto update. }\end{array}$ & 1. & $\begin{array}{l}\text { Use for a stand-alone } \\
\text { system. }\end{array}$ \\
\hline BACnet-based FDS & 2014 & 1. & $\begin{array}{l}\text { Monitoring system } \\
\text { using MS/TP } \\
\text { protocol. } \\
\text { reference model } \\
\text { is operated by } \\
\text { wireless- } \\
\text { BACnet for } \\
\text { flexibility. }\end{array}$ & 2. & $\begin{array}{l}\text { Signal is directly } \\
\text { dependent upon the } \\
\text { traffic load. } \\
\text { Inter-operability of } \\
\text { different vendors' } \\
\text { field devices makes } \\
\text { problems. }\end{array}$ \\
\hline Addressable FACS & 2015 & 1. & $\begin{array}{l}\text { Broad range of } \\
\text { device connecting } \\
\text { capabilities. }\end{array}$ & 1. & $\begin{array}{l}\text { Standard capacity } \\
\text { of maximum } 127 \\
\text { points. }\end{array}$ \\
\hline $\begin{array}{l}\text { Dedicated Computer } \\
\text { Controlled system }\end{array}$ & 2016 & 1. & $\begin{array}{l}\text { Added power, } \\
\text { Internet } \\
\text { connectivity, and } \\
\text { remote service } \\
\text { capabilities }\end{array}$ & $\begin{array}{l}1 . \\
2 .\end{array}$ & $\begin{array}{l}\text { Failure of dedicated } \\
\text { computer or } \\
\text { connection problem } \\
\text { can fail the system. } \\
\text { Required } \\
\text { maintenance. }\end{array}$ \\
\hline $\begin{array}{c}\text { Address-able VAD } \\
\text { (Visual Alarm Device) }\end{array}$ & 2010 & 1. & $\begin{array}{l}\text { Visual Alarm } \\
\text { Device. }\end{array}$ & $\begin{array}{l}1 . \\
2 .\end{array}$ & $\begin{array}{l}\text { For a small plant. } \\
\text { Extension not } \\
\text { possible. }\end{array}$ \\
\hline $\begin{array}{l}\text { Pattern matching } \\
\text { process }\end{array}$ & 2015 & 1. & $\begin{array}{l}\text { Use predefined } \\
\text { pattern matching } \\
\text { process. } \\
\text { Signature-based } \\
\text { traffic. }\end{array}$ & 1. & $\begin{array}{l}\text { The performance } \\
\text { depends on detection } \\
\text { engine. }\end{array}$ \\
\hline Mechatronic approach & 2016 & 1. & $\begin{array}{l}\text { Autonomous } \\
\text { fire-suppression } \\
\text { module was used. } \\
\text { Versatile device } \\
\text { addable. }\end{array}$ & 1. & $\begin{array}{l}\text { The autonomous } \\
\text { system used is } \\
\text { expensive. }\end{array}$ \\
\hline Optical fibre sensors & 2014 & $\begin{array}{l}1 . \\
2 .\end{array}$ & $\begin{array}{l}\text { Long durability. } \\
\text { Long distance } \\
\text { detection. }\end{array}$ & 1. & $\begin{array}{l}\text { The problem of hot } \\
\text { air. }\end{array}$ \\
\hline
\end{tabular}




\section{Research Method}

The proposed system is a combination of successive interference cancellation (SIC) and Minimum Mean Square Error (MMSE) or can be written as SIC-MMSE. In this process, initially, the raw data is sampled and prepared for sub-band packaging according to users' data symbol. The coder is joined along with MMSE detection system, which will depend upon user or operator. The MMSE detection process will continue to do channel pilot sensing, testing signal quality, estimate the Signal to Noise Ratio (SNR), arrange Channel Matrix (CM) formation, Channel selection \& estimation. The MMSE processed data will be filtered for maximum correlation detection, which is the part of SIC method. This method is used to detect the sequentially processed data according to the users' symbols and regenerate the data to transfer it through the new channel. While MMSE will constantly monitor the signal quality to realter the channel coefficient, the SIC will help MMSE to improve its performance by processing sequential data so that MMSE can reselect any parameters at any moment to reduce interruption and data loss. At the end of transmission process, the RF modulation will modulate the data then filter with Spectrum Shaping Filter (SSF) and transmit through the channel. A synchronizer is used in transmission process to synchronize any disrupted operation. On the receiver side the signal will be demodulated and reshaped with SSF. After demodulation the same concept of proposed MMSE will be used to decode the data. The synchronizer on the receiver side and transmitter side will be synchronized together through MMSE. Finally, the decoded data will be reframed using same SIC method. This combination (SIC-MMSE) can reduce the channel shortage and performance losses.

\subsection{Symbolize Error Vector}

A multiple user with multiple nodes for Base Station (BS) was considered based on Time Division Duplex (TDD) method where upload and download channel data links consider within coherence interval in a point to point MIMO system. Considering the system have N numbers of nodes on a base station per cells having $M$ number of total antennas per cells and $\mathrm{K}$ number of single antennae known as User Terminal (UT) in each cell. For K antenna user terminal to base station $\mathrm{J}$ can be expressed as:

$$
\mathrm{H}_{j k}=\mathrm{B}_{j k} \mathrm{G}_{j k}
$$

where, $\mathrm{H}_{j k}$ is the fading's on $\mathrm{J}$ station for $\mathrm{K}$ number of single antennae. $B_{j k}$ is the fading coefficient of large scale and $\mathrm{G}_{j k}$ is the fading coefficient of small scale. Here, $\mathrm{B}_{j k}$ represent path loss and shadow fading of the channel. The matrix was denoted by upper case and bold uppercase used for vector identifications. The $\mathrm{G}_{j k}$ is the total nodal fading effect induced in per cell's capacity can be represented by ${ }^{7}$,

$$
\mathrm{G}_{j k}=\mathrm{CM}\left(0, \mathrm{I}_{m}\right)
$$

Where, $\mathrm{C}$ and $\mathrm{M}$ are the capacity sum rate \& number of BS antennas respectively and $\mathrm{I}_{m}$ is the indication function of M. So,

$$
\mathrm{H}=\mathrm{G}
$$

Here, $\mathrm{H}$ is channel fading, $\mathrm{B}$ represents the largescale diagonal matrix and $G$ represents the small-scale matrix each column represents a channel from UT to BS ${ }^{8}$. When the number of BS antennas increase the channel, the approximates orthogonal matrix will be $=\mathrm{B}$. Each terminal is assigned with a pilot sensing for $\mathrm{k}$ number of single antennas, the sensing pilot $s_{k}$ with power equal to, $\mathrm{s}_{\mathrm{k}}, \mathrm{t}=\left[\mathrm{s}_{\mathrm{k} 1} ; \mathrm{s}_{\mathrm{k} 2} \ldots \mathrm{s}_{\mathrm{kt}}\right] \mathrm{T}$ and at each BS station, ${ }_{2}=0$, if $\mathrm{j} \neq \mathrm{k}$ and the transmitted power is equal for all pilots. For the conventional detection the receiver vector matrix y can be denoted by,

$$
y \in C^{M \times 1}[8] \text { or } y=H x+n
$$

where, $\mathrm{C}$ is channel matrix and complex additive white gaussian noise (AWGN) vector, $\mathrm{H} \in \mathrm{C}^{\mathrm{M} \times \mathrm{K}}, \mathrm{x}$ is the symbol vector sent by $\mathrm{K}$ user can be denote by $\mathrm{x} \in \mathrm{C}^{\mathrm{k} \times 1}$ and number of nodes $n$. If the symbol error vector $e$ then, 


$$
\mathrm{e}=\mathrm{x}-\dot{\mathrm{x}}
$$

Here, $\dot{\mathrm{x}}$ is the receiving signal. Assuming correlation parameter $\sigma$ is known perfectly at the base stations and $\mathrm{h}[\mathrm{n}]$ be the channel vector between a UT and a BS at time t. Then ${ }^{9}$,

$$
h[t]=\sigma h[t-1]+e v[t]
$$

Here, $t$ is time index and $\mathrm{e}[t]$ is white noise with zero mean and temporal correlation parameter $\sigma^{2}=((1-2))$ obtained through the Yule-Walker equation ${ }^{7}$. The channel model above is known as the stationary ergodic GaussMarkov block fading channel model ${ }^{8}$.

\subsection{MMSE Detection Process}

For the MIMO model equation according to reference $\mathrm{no}^{7}$, where receiving signal vector $\hat{y}$ from receiver signal $\mathrm{y}$ and the fibrinous norm ${ }_{2}$ to limit sphere of validity of general norm.

$$
\hat{y}=y-H \dot{x}=H(e+x)
$$

where, $\mathrm{x}$ is transmitted symbol massages and $\dot{\mathrm{x}}$ is the received symbol massages. Error vector e should be zero for ideal communication system. So, that the error detection should be overcome from receiver signal vector. Some researcher expresses the compressing sensing methods, where they proposed to naturally consider the symbol error vector $e^{7}$. In compressing sensing methods $M$ should be less then $K$, but if $M$ becomes more then equal to $\mathrm{K}$, this system will be impractical. For MIMO multi-antenna mode, the $\mathrm{M}$ is generally greater then equal $\mathrm{K}$, the receiver signal vector later filter by matrix $\mathrm{W}_{\text {MMSE }}$ is given by the equation,

$$
\mathrm{W}_{\mathrm{MMSE}}=\frac{H^{h}}{H H^{h}+I_{m}}
$$

where, $\mathrm{W}$ is a predefine filter matrix, $\mathrm{W}_{\mathrm{MMSE}}$ is the filter matrix for MMSE matrix for and AWGN (Gaussian noise) vector $\mathrm{n} \in \mathrm{CM}$ for $\mathrm{CM}\left(0, \mathrm{I}_{m}\right)^{8}$. By Maximum $\mathrm{A}$
Posterior (MAP) detection known as detection system detection method the optimal detection é can be found from the reference paper no ${ }^{10}$.

$$
\mathrm{e} \cong \underset{\in \in \mathrm{A}^{\mathrm{A}} \mathrm{A}}{\arg \max }\left(1 / \sqrt{2 \pi \sigma^{2}} \exp \frac{-0.707}{\sigma^{2}}\left(\left(\left\|\left.\right|^{\wedge} \mathrm{y}-\mathrm{He}\right\|\right)_{2}^{2} \operatorname{Pr}(\mathrm{e})\right.\right.
$$

According to the paper the approximation is because of e and $n$ dependency and may omit while SNR increases and can be precise at high SNRs. $\operatorname{Pr}(\mathrm{e})$ is probability of priority error symbol. When BPKS values are $+1 \&-1$, is the finite alphabet having the values $-2,0 \&+2$ and for the nonzero value of detection error becomes - 2 \& 2 . If transmitted symbols are from -1 to 1 , then the possibility of the e will be no zeroes from +2 to -2 and possible probability can be $0.5 P$. when $\lambda$ is the degree of sparsity, $=0.25$. If, e is the element of and $e^{\circ}$ is the symbol error vector for initial iteration, by solving the equation no (5)

$$
\begin{aligned}
\mathrm{e}^{\circ} & =\frac{H^{H \varkappa} \mathrm{y}}{H H^{H}+0.5 \lambda} \quad ;\left[\text { if, is finite and initially é }=\mathrm{e}^{\circ}\right] \\
\mathrm{e}^{\circ} & =M^{\mathrm{n}} \mathrm{y} \quad
\end{aligned}
$$

Here, $M$ is MMSE detection method with tuneable degree of spares $\lambda$, where, $\lambda$ is the replacement of noise. If, $\mathrm{Q} \theta\left({ }^{*}\right)$ is vector dividing function and $\theta$ optimal threshold then, optimal detection, $\mathrm{e}^{=} \mathrm{Q} \theta\left(\mathrm{e}^{\circ}\right)$ for discrete function. So, we can rewrite the equation

$$
\mathrm{e}^{\prime}=\mathrm{Q} \theta\left(\mathrm{e}^{\circ}\right)=2 \sin \left(\mathrm{e}^{\circ}\right) \mathrm{I} ;\left[\left\|\mathrm{e}^{\mathrm{o}}\right\|>\theta\right]
$$

where, $I$ is an indication functions. If, the optimal threshold, $\theta=\left\{\theta_{1}, \theta_{2}, \theta_{3} \ldots . \theta_{n}\right\}$ and for the non-zero components, $\mathrm{e}=\{0, \pm 2\}$; [i.e. $\|\mathrm{e}\|<\theta$ ]. Similarly, QPSK detection the equivalent transform with real $(R)$ and imaginary (I), where $\mathrm{I}(\mathrm{e})$ and $\mathrm{R}(\mathrm{e})$ parts of $\mathrm{x}$,

$$
\text { é }=2 \sin [\{\mathrm{R}(\mathrm{e})+\mathrm{I}(\mathrm{e})\} \mathrm{T}] ; \quad\left[\text { Where, } \mathrm{e}^{\circ} \in\right]
$$


Here, $y^{\circ}$ initial receiver signals, $n$ is the Gaussian noise, $\mathrm{e}(l)$ is the $l^{\text {th }}$ symbol error vector. The equation (8) is the prior probability detection of e. If, $\mathrm{e}(l)$ is non-zero, for the $n^{\text {th }}$ entry, $x_{m}^{l-1}$ is of $x^{l-1}$, the for $n^{\text {th }}$ entry of $l^{\text {th }}$ symbol,

$$
y_{n}^{\circ l}=\mathrm{W}(1-1) e_{n}^{l}+\sum_{i \neq j} w_{n j}^{1-l} e_{j}^{l}+x_{n}^{l-1}
$$

So, Gaussian approximates with following variance $\sigma$,

$$
\left(\sigma_{n}^{l-1}\right)^{2}=\sum_{n \neq j}^{n} 4\left(\omega_{j n}^{1-l}\right)^{2} p^{l-1}+\left\{\sum_{n}(l-1)\right\}
$$

The error probability for $n^{\text {th }}$ entry can be solving in same way from the optimal detection equation no 5 . If the operator for MMSE is $\mathrm{Q}$ for the sensing pilot $\mathrm{s}_{\mathrm{k}}$ is given by the equation,

$$
\mathrm{s}_{\mathrm{k} i}=\mathrm{Q}\left(y_{\mathrm{k} i}\right)=\mathrm{Q}\left(g_{\mathrm{k} i} r_{i}\right)
$$

Here, $y_{k}$ is the output of $k$ antenna, the chosen vector $g_{\mathrm{k}}$ and $\mathrm{r}$ is the degree of freedom. The operator Q can be used to give instruction where the sampling should stop for a transmission. The ideal analysed threshold can minimize the probability of error by $\frac{d}{d x} p_{n}^{l}=0$. If, the optimal threshold for $n^{\text {th }}$ entry of é is can be found from the equation no 11,

$$
\theta_{n}^{i}=w_{m n}^{1-l}+\frac{1}{2}\left\{\frac{\lambda}{\omega_{n n}^{-1}}\right\}^{l-1} \cdot\left(\sigma_{n}^{l-1}\right)^{2}
$$

From the above equations, the proposed MMSE detector can be iteratively exploited to improve the detection performance. To minimize the errors, it is possible to refit the tuneable parameters like degree of sparsity $(\lambda)$, selection of different channel based on lower SNR or MSE and availability of CM.

\subsection{Algorithm}

Considering the mm-wave MIMO system with Distributed Antenna System (DAS) configuration, where, number of base antenna $M_{B}$ having $\mathrm{k}$ number of single antenna and $\mathrm{N}$ number remote radio heads. If the $\mathrm{Q}$ user also equipped with $M_{U}$ antenna, the receiving anten-

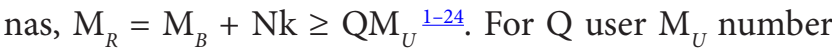
flat fading channels, the MMSE pilot $s_{k}$ was considered before now can be rewritten as vectoral form, $\mathrm{s}_{k} \in \mathrm{C}_{u}^{\mathrm{M} \times 1}$. From the model as Gauss-Markov block fading channel shown above in equation no.3, the data vector $\mathrm{s}_{k}$ have zero mean.

The SIC algorithm relies on sequential detection receiver signals, where it is required to equalize the channel matrices $\mathrm{W}_{\text {MMSE }}$ given in equation no. 4 , then carrier channels can get the higher Signal to Interference Noise Ratio (SINR). From the reference no ${ }^{27}$ the SINR per symbol for $I^{\text {th }}$ iteration for the $j^{\text {th }}$ number of symbols is thus can be expressed as,

$$
\operatorname{SINR}_{j}^{i}=\left(\sigma^{i}\right)^{-2}\left(a^{i}\right)^{2}\left(\left|s_{k i}\right|\right)^{2}
$$

where, is the amplitude, Gaussian approximates variance, pilot $\mathrm{s}_{k}$ for $I^{\text {th }}$ iteration. The fading matrix $\mathrm{H}_{k}$ for $\mathrm{k}$ user, having $\mathrm{N}+1$ submatrix in each remote radio head, then, $\mathrm{H}_{\mathrm{k}}=\left[\mathrm{H}_{\mathrm{kl}}, \mathrm{H}_{\mathrm{k} 2}, \ldots . . \mathrm{H}_{\mathrm{k}(\mathrm{N}+1)}\right]^{\mathrm{T}}$. When the symbol is deciding according a decision will be made depends on MMSE operator given in equation no. 11. Instead of executing don't care sign decision, it is possible to use operator Q as soft switch through the hyperbolic tangent non-linear detector whose argument is weighted by an estimation of the SINR ${ }^{25}$. So the expression for $\mathrm{s}_{\mathrm{k}}$ for $I^{\text {th }}$ iteration can be given in QPSK constellation as,

$s_{k i}=0.707\left[\tanh \left\{R\left(y_{k i}\right) /\left(\sigma_{k i}^{2}\right)\right\}+j \tanh \left\{I\left(y_{k i}\right) /\left(\sigma_{k i}^{2}\right)\right\}\right]$

Finally, for the decoded case in receiver end, while all symbols are retrieved, the don't care decision will perform for the resulting output $y=\left(y_{1}, y_{2}, \ldots ., y_{n}\right)^{T}$. 


\section{Measurement and Simulations}

For MATLAB simulation we used Gaussian noise as reference with different SNR levels to analyse the performance of the proposed SIC-MMSE system. In this simulation process we have compared results with conventional mm-wave MIMO system and MMSE system. For the simulation process we first considered the number of antennae per cells $M=1000$. For the process, initially we detected symbol vector $\mathrm{j}$ using conventional MIMO system and proposed MMSE. For the output SNRs priority probability for conventional and MMSE we followed equations $\frac{7-10}{}$.

$$
\begin{aligned}
& \lim _{S N R \rightarrow \infty} \frac{\log P_{c}(S N R)}{\log S N R}=-d ; \\
& \lim _{S N R \rightarrow \infty} \frac{\log P_{S I C-M M S E}(S N R)}{\log S N R}=-d
\end{aligned}
$$

Then, the degree of sparsity $\lambda$, can be obtained from $\lambda=\ln \left[\frac{2(1-p)}{p}\right]$; Considering the MMSE linear detection, for the $I^{\text {th }}$ iteration the error probability é for SIC-MMSE based MIMO was obtained from equation no. 8, where optimal threshold $\theta_{m}^{l}$ was obtained by solving the equation no. 7. This proposed research was conduct on Time Division Duplex (TDD) method. So, to determine the Spectral Efficiency (SE) for SIC-MMSE is expressed ${ }^{25}$ :

$$
\eta_{\text {hMMSE }}=\frac{\left(T_{f}-T_{p}-T_{t}\right) N_{i}}{T_{i} N_{s}}
$$

where, $\mathrm{Tp}=$ preamble period, $\mathrm{Tt}=$ trailer time period, $\mathrm{Tf}=$ frame duration and $\mathrm{Ns}=$ number of symbols in a $\mathrm{t}$ time slot, $\mathrm{Ni}=$ number of information bits. By resolving

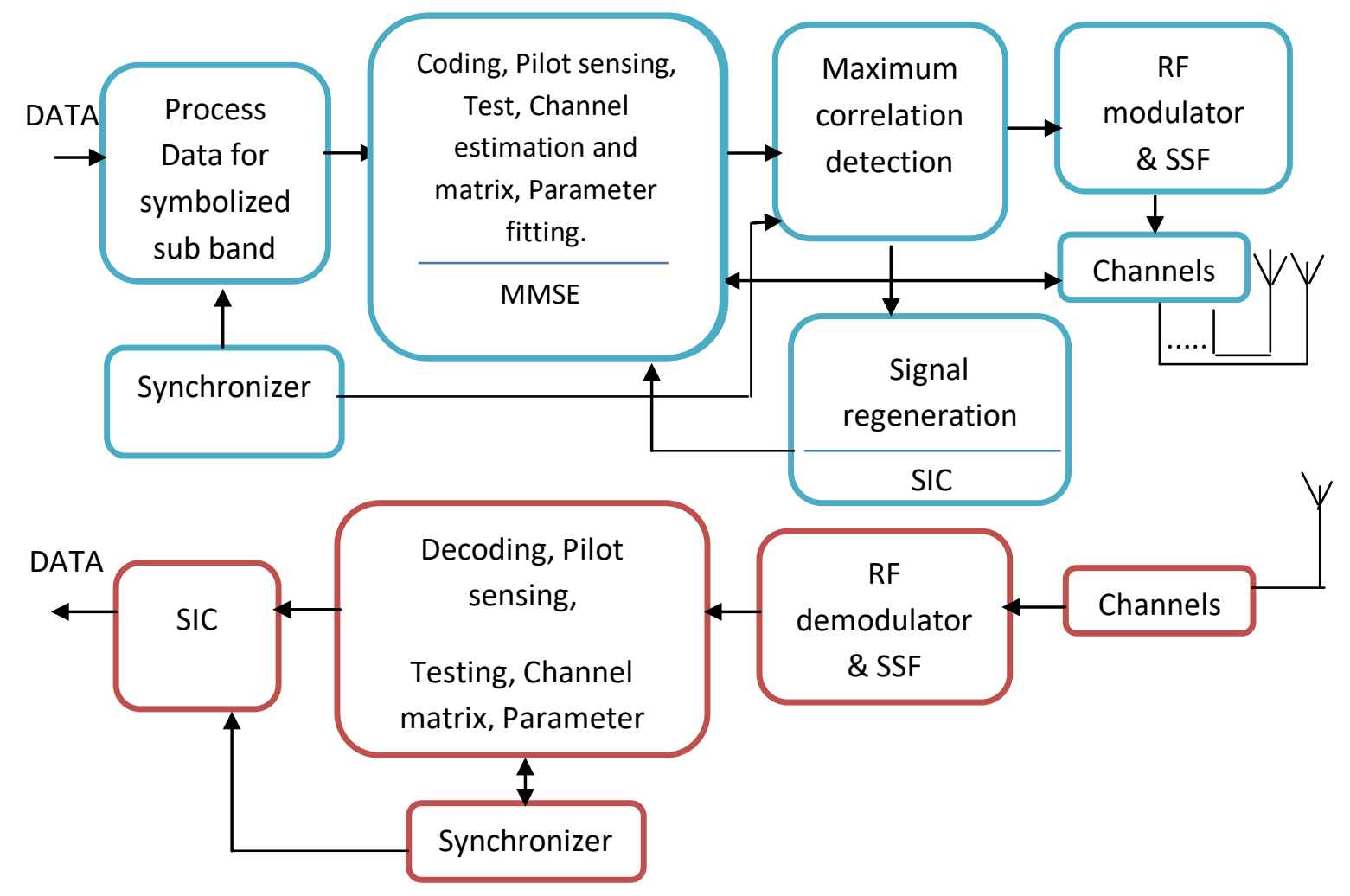

Figure 1. Proposed system approximation for transmission and receiver. 


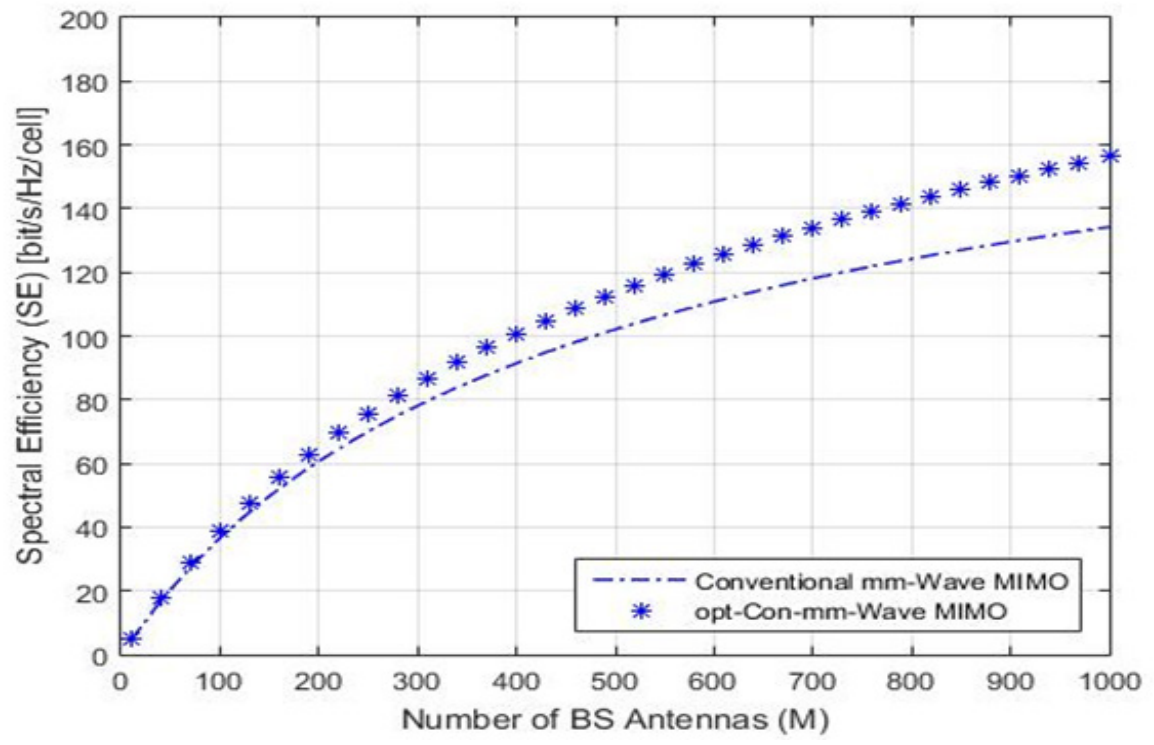

Figure 2. Conventional mm-waves MIMO and optimized mm-waves MIMO spectral efficiency performance with the increase number of Antennas.

the equations in MATLAB finally we got SE for the SICMMSE.

Simulating the proposed system in MATLAB the performance of SIC-MMSE was achieved. For the comparison and benchmarking we also simulated the conventional mm-Wave. Where the simulation was done by Spectral Efficiency (SE) [bit/s/Hz/cell] vs Number of BS Antennas (M).

The Figures 1 and 2 shows the comparison of the Spectral Efficiency (SE) with the increase number of BS

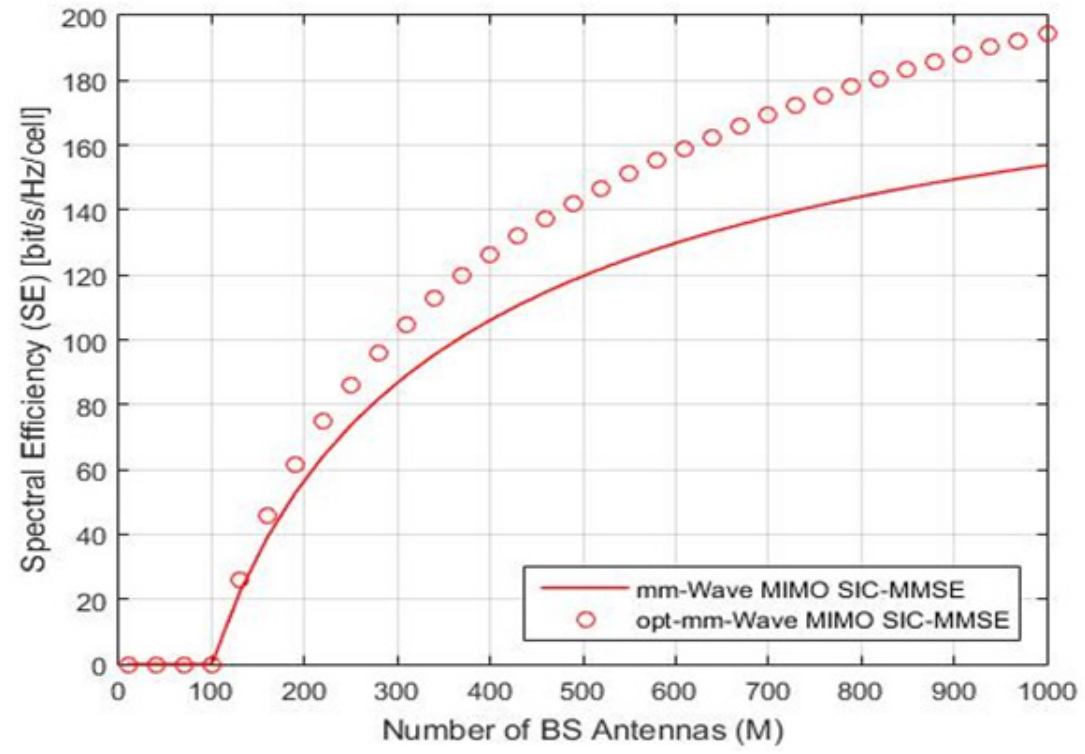

Figure 3. Proposed SIC-MMSE and optimized SIC-MMSE spectral efficiency performance according to increase number of Antennas. 


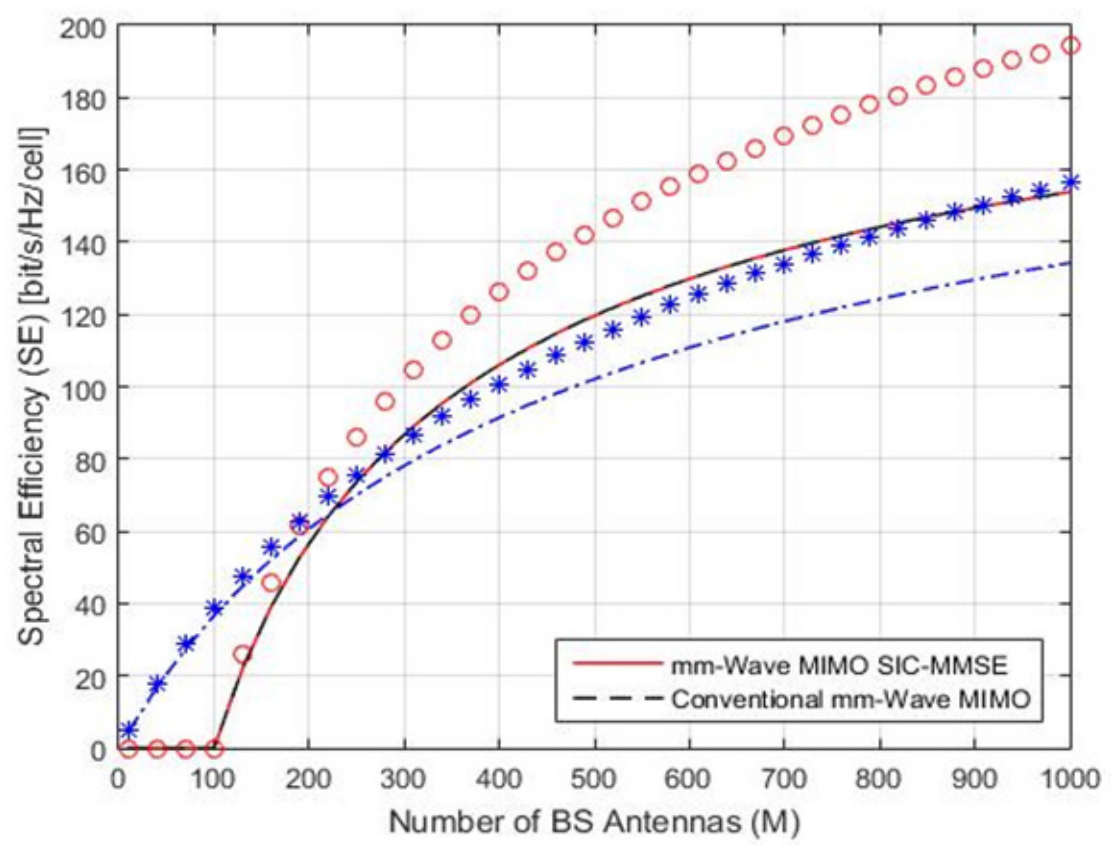

Figure 4. The performance of both methods' spectral efficiency according to increase number of antennas.

antenna at base station for conventional or single millimetre-Wave system. Where it is depicted that spectral efficiency increases from 0 to maximum $136 \mathrm{bits} / \mathrm{s} / \mathrm{Hz} /$ cell with the increase of base antennas from 0 to 1000 . Where, the parameters are optimized for the better performance, the maximum SE was recorded to $157 \mathrm{bits} / \mathrm{s} /$ $\mathrm{Hz} /$ cell for the 1000 number of antennas.

The SIC- MMSE simulation in Figure 3 shows better performance than conventional mm-Wave MIMO system after optimization. Before optimization the maximum SE was found to $133 \sim 132 \mathrm{bits} / \mathrm{s} / \mathrm{Hz} / \mathrm{cell}$ while number of antennas was maximum. Where, after optimization the value crossed 195 bits/s/Hz/cell. Every system requires optimization, where without optimization this proposed system performed almost the equal to the optimized conventional mm-wave MIMO system, but after optimization it increased rapidly. Figure 4 shows the performance comparison simulation block for the both methods having same parameters, spectral efficiency according to increase number of antennas.

\section{Conclusion and Future Work}

This paper has presented a communication method which is the combined methodology of MMSE and SIC technique for mm-Wave MIMO based wireless communication system. The combined method was proposed to reduce the relative orthogonal coordination and multiple node detection problems while transmitter or receiver moves. The development of the equations was done by comparing, reading and reoptimizing the existed several concepts. From the simulation it can found that, the proposed combined technique for wireless power communication is better than conventional mm-wave MIMO. Though, the Proposed SIC-MMSE require optimization for better performance more combined technique with better optimization can lead a better performance then single one. In future we would like to improve this research by adding more system together for optimal performance and compare with recent research. 


\section{References}

1. Aalto University, AT\&T, BUPT, CC, Ericsson, Huawei, Intel, KT Corporation, Nokia, NTT DOCOMO, NYU, Qualcomm, Samsung, U. Bristol, and USC. White paper on '5G channel model for bands up to $100 \mathrm{GHz} ; 2016$.

2. Sun S, Rappaport TS, Thomas TA, Ghosh A, Nguyen HC, Kava's IZ, Rodriguez I, Koymen OH, Partyka A. Investigation of prediction accuracy, sensitivity, and parameter stability of large-scale propagation path loss models for $5 \mathrm{G}$ wireless communications. IEEE Transactions on Vehicular Technology. 2016; 65(5):2843-60. https://doi. org/10.1109/TVT.2016.2543139.

3. Raghavan V, Partyka A, Akhoondzadeh-Asl L, Tassoudji MA, Koymen $\mathrm{OH}$, Sanelli J. Millimeter wave channel measurements and implications for PHY layer design. IEEE Transactions on Antennas and Propagation. 2017; 65(12):6521-33. https://doi.org/10.1109/ TAP.2017.2758198.

4. Rusek F, Persson D, Lau BK, Larsson EG, Marzetta TL, Edfors O, Tufvesson F. Scaling up MIMO: Opportunities and challenges with very large arrays. IEEE Signal Processing Magazine. 2013; 30(1):40-60. https://doi.org/10.1109/ MSP.2011.2178495.

5. Roh W, Seol JY, Park J, Lee B, Lee J, Kim Y, Cho J, Cheun K, Aryanfar F. Millimeter-wave beamforming as an enabling technology for $5 \mathrm{G}$ cellular communications: Theoretical feasibility and prototype results. IEEE Communications Magazine. 2014; 52(2):106-13. https://doi.org/10.1109/ MCOM.2014.6736750.

6. Directional beam forming for millimetre wave MIMO systems [Internet]. [cited 2016 Nov 11]. Available from: http:// www.arxiv.org/abs/1601.02380.

7. Anderson CR, Rappaport TS. In-building wideband partition loss measurements at 2.5 and $60 \mathrm{GHz}$. IEEE Transactions on Wireless Communications. 2004; 3(3):1-7. https://doi. org/10.1109/TWC.2004.826328.

8. Raghavan V, Cezanne J, Subramanian S, Sampath A, Koymen $\mathrm{OH}$. Beam forming trade offs for initial UE discovery in millimeter-wave MIMO systems. IEEE Journal of Selected Topics in Signal Processing. 2016; 10(3):543-59.

9. Sun S, Rappaport TS, Heath RW, Nix A, Rangan S. MIMO for millimeter wave wireless communications: Beamforming, spatial multiplexing, or both? IEEE Communications Magazine. 2014; 52(12):110-21. https://doi.org/10.1109/ MCOM.2014.6979962.

10. Raghavan V, Subramanian S, Cezanne J, Sampath A, Koymen OH, Li J. Directional hybrid precoding in millimeter-wave MIMO systems. IEEE Global Communications
Conference; 2016. p. 1-7. https://doi.org/10.1109/ GLOCOM.2016.7841735.

11. Ran R, Wang J, Oh SK, Hong SN. Sparse-aware minimum mean square error detector for MIMO systems. IEEE Communications Letters. 2017; 21(10):2214-17. https:// doi.org/10.1109/LCOMM.2017.2723362.

12. Raghavan V, Subramanian S, Cezanne J, Sampath A, Koymen OH, Li J. Single-user vs. multi-user precoding for millimeter wave MIMO systems. IEEE Journal on Selected Areas in Communications. 2017; 35(6):1387-401. https:// doi.org/10.1109/JSAC.2017.2687798.

13. Li A, Masouros C. Hybrid precoding and combining design for millimeter-wave multi-user MIMO based on SVD. IEEE International Conference on Communications; 2017. p. 1-6. https://doi.org/10.1109/ICC.2017.7996970.

14. El Ayach O, Rajagopal S, Abu-Surra S, Pi Z, Heath RW. Spatially sparse precoding in millimeter wave MIMO systems. IEEE Transaction Wireless Communication. 2014; 13(3):1499-513. https://doi.org/10.1109/ TWC.2014.011714.130846.

15. Alkhateeb A, El Ayach O, Leus G, Heath RW. Channel estimation and hybrid precoding for millimeter wave cellular systems. IEEE Journal of Selected Topics in Signal Processing. 2014; 8(5):831-46.

https://doi.org/10.1109/JSTSP.2014.2334278.

16. Alkhateeb A, Leus G, Heath RW. Limited feedback hybrid precoding for multi-user millimeter wave systems. IEEE Transactions on Wireless Communications. 2015; 14(11):6481-494. https://doi.org/10.1109/ TWC.2015.2455980.

17. Sohrabi F, W. Yu. Hybrid digital and analog beamforming design for large-scale antenna arrays. IEEE Journal of Selected Topics in Signal Processing. 2016; 10(3):501-13. https://doi.org/10.1109/JSTSP.2016.2520912.

18. Sadeghi M, Bjornson E, Larsson EG, Yuen C, Marzetta TL. Max-min fair transmit precoding for multi-group multicasting in massive MIMO. IEEE Transactions on Wireless Communications. 2018; 17(2):1358-73. https://doi. org/10.1109/TWC.2017.2777987.

19. Noh S, Zoltowski MD, Love DJ. Training sequence design for feedback assisted hybrid beamforming in massive MIMO systems. IEEE Transactions on Communications. 2016; 64(1):187-200. https://doi.org/10.1109/ TCOMM.2015.2498184.

20. Cellular communications system with centralized base stations and distributed antenna units [Internet]. [cited 1997]. Available from: https://patents.google.com/patent/ US5657374. 
21. Distributed antenna systems [Internet]. [cited 2018 Nov 05]. Available from: https://en.wikipedia.org/wiki/Distributed_ antenna_system.

22. Roh W, Paulraj A. MIMO channel capacity for the distributed antenna. Proceedings IEEE 56th Vehicular Technology Conference. 2002; 2:706-9.

23. Zhuang H, Dai L, Xiao L, Yao Y. Spectral efficiency of distributed antenna system with random antenna layout. Electronic Letters. 2003; 39(6):495-6. https://doi. org/10.1049/el:20030327.
24. Castanheira D, Gameiro A. Distributed antenns systems caacity scaling [coordinated and distributed MIMO]. IEEE Wireless Communications. 2010; 17(3):68-75. https://doi. org/10.1109/MWC.2010.5490981.

25. Debbah M, Muquet B, De Courville M, Muck M, Simoens S, Loubaton P. A MMSE successive interference cancellation scheme for a new adjustable hybrid spread OFDM system. IEEE Transactions on Vehicular Technology. 2000; 2:745-9. 